\title{
HRTEM Contrast Analysis for Structure Characterization of Graphene Films Grown by CVD
}

\author{
P. Plachinda*, S. Rouvimov ${ }^{*}$, R. Solanki*, K. Langworthy ${ }^{* *}$ \\ *Department of Physics, Portland State University, Portland, OR 97207-0751 \\ ${ }^{* *}$ CAMCOR, University of Oregon, Eugene, OR
}

Low voltage $(80 \mathrm{kV})$ aberration corrected high-resolution transmission electron microscopy (HRTEM) appeared to be the most promising technique for structure characterization of graphene [1], enabling a direct observation of defects [2], impurities, etc. However, due to low contrast and special imaging conditions [3] the contrast interpretation still requires computer simulations for reliable structure identification. This paper addresses the HRTEM contrast of graphene films as a function of number of the layers, their stacking sequence, presence of defects and imaging conditions based on multislice contrast simulations.

Graphene films were grown on the Ni substrates [4] which were transferred to copper grids for electron microscopy analysis. FEI's Titan microscope (equipped with Cs-corrector) operated at $80 \mathrm{kV}$ was employed to view the graphene films at atomic resolution. Figure 1 shows experimental HRTEM images of a graphene film near and at the edge. The contrast simulations shown in Figs. 2-4 demonstrate that the contrast symmetry may provide useful information about the atomic arrangement in crystal lattice of graphene film. The contrast symmetry is sensitive to stacking sequence (Fig. 2), atomic configuration at the edge (Fig. 3), and position of a foreign atom in the crystalline lattice (Fig. 4), and thus, provides a pathway to analyze the structure of the graphene films.

We propose compiling the "HRTEM contrast library" based on the computer simulations of contrast from various types of defects and possible structural configurations that can be used for "fingerprinting" of structure features and defects in graphene. This includes the AA and the $\mathrm{AB}$ stacking in graphene, adatoms in various positions, and the "zig-zag" and "armchair" ribbons, etc. Such "library" would allow monitoring of the number of graphene layers, the atomic configuration at the edges (zig-zag versus armchair) and lattice defects, and the location of adsorbed impurities on the graphene crystal lattice. This paper also addresses the importance of the aberration corrected microscopy for investigation of graphene and especially defects in graphene films.

The research was supported by the Oregon Nanoscience and Microtechnologies Institute. One of the authors (SR) also acknowledges the support by the National Center for Electron Microscopy (NCEM) at LBNL, Berkeley.

[1] K. S. Novoselov, A. K. Geim, S. V. Morozov, et al, Science, 306, (2004),666.

[2] Warner, J. H., Rümmeli, M. H., Gemming, et al. Nano Letters, 9(1) (2008)

[3] J. C. Meyer, C. Kisielowski, R. Erni, et al, Nano Lett., 8, (2008), 3582-3586

[4] G. Nandamuri, S. Roumimov, and R. Solanki, Nanotechnology 21, (2010), 145604 

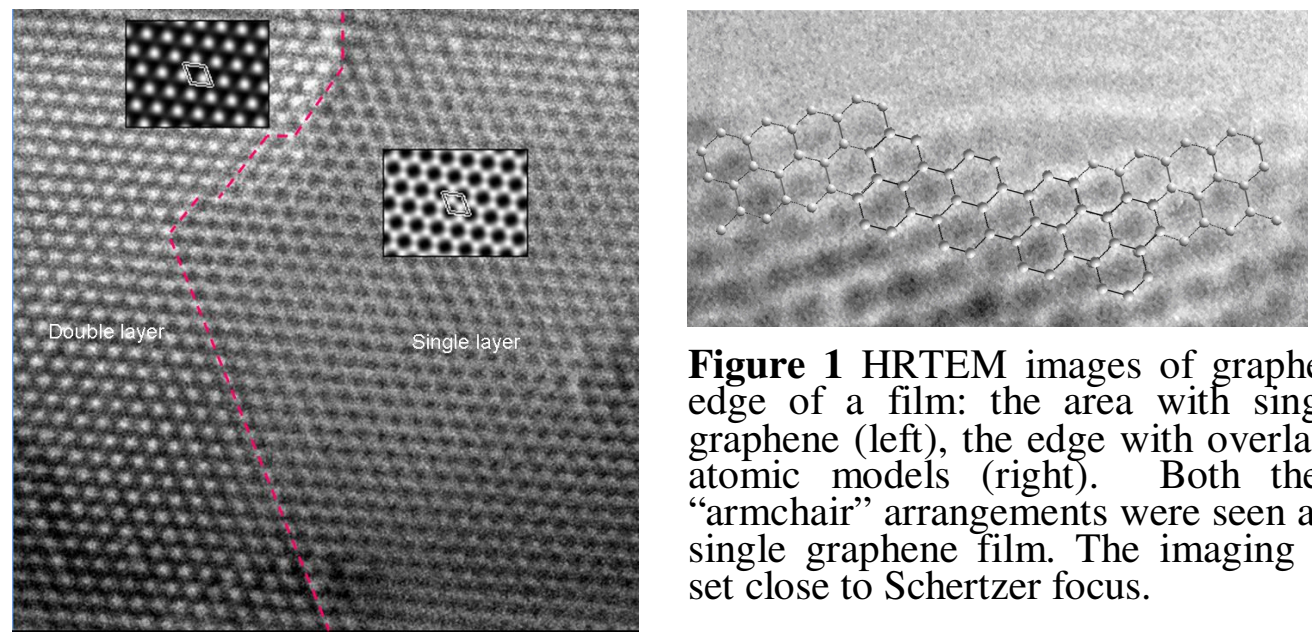

Figure 1 HRTEM images of graphene taken at the edge of a film: the area with single and bi-layer graphene (left), the edge with overlaid stick-and-ball atomic models (right). Both the "zigzag" and "armchair" arrangements were seen at the edge of the single graphene film. The imaging conditions were set close to Schertzer focus.

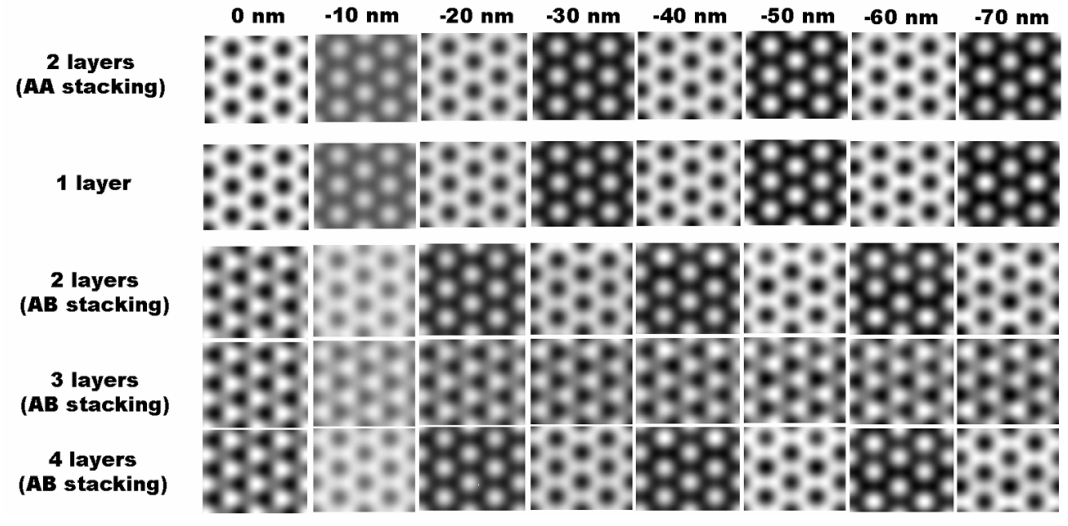

Figure 2 Computer simulations of HRTEM contrast of single- and bilayer graphene (the first and second rows, respectively) at defoci of 0 and $-60 \mathrm{~nm}$ (the left and right columns, respectively). Note: Contrast on the simulated images has been maximized and therefore, looks much higher as compared to experimental images of Fig. 1.

Figure 3. Computer simulations of HRTEM contrast of the "armchair" (top) and the "zigzag" (bottom) ribbons of single graphene layers. Imaging conditions: $\mathrm{U}=80 \mathrm{kV}, \mathrm{Cs}=0, \mathrm{Cc}=0$, defocus values are shown on the left. Note: The contrast is strongly influenced by the Fresnel-fringes.
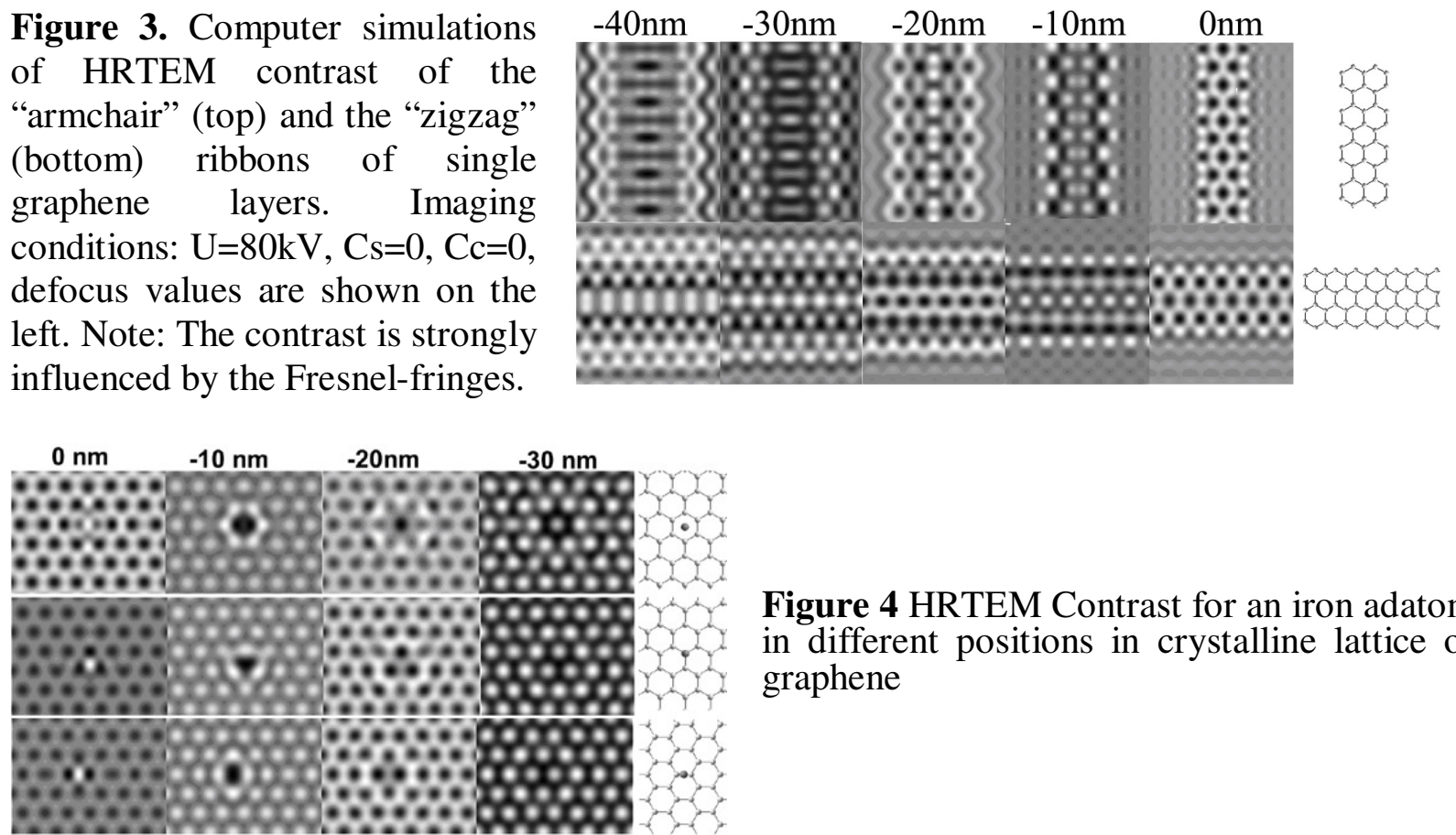

Figure 4 HRTEM Contrast for an iron adatom in different positions in crystalline lattice of graphene 